\title{
Fuel temperature estimation and energy balance within an UAV integral wing fuel tank
}

\author{
P. Blázquez \\ Airbus Defence and Space, Spain
}

\begin{abstract}
The endurance of Unmanned Air Vehicles (UAVs) is an essential driver for the design and development of such vehicles. Medium altitude long endurance air vehicles (MALEs) are designed to perform different missions for long periods of time in different altitudes from sea level up to $36 \mathrm{kft}$. Endurance can be improved by increasing the fuel content and designing high aspect ratio wings to increase the aerodynamic performances.

The integral wing fuel tank is defined by the upper and lower skins and the front and rear spars. The only insulation from external air is the skin thickness. On the other hand, wings with a high aspect ratio indicate long and narrow wings resulting in a high surface-to-volume ratio. These two factors lead to a high heat flux between the fuel and external air.

Dynamic estimations of fuel temperature along different aircraft missions are needed to evaluate the different fuel requirements (i.e. freezing, fuel injection, cooling, etc.) from the beginning of the project.

The present paper describes a dynamic model of a wing fuel tank. It is focused on validating the wing fuel system design from a thermal point of view. It estimates the temperatures and energy balance of the wing fuel along the missions taking into consideration the wing geometry, the different heat transfer effects, as well as the flight conditions and engine fuel consumption.

Keywords: simulation, aircraft, fuel, heat transfer, freezing, cooling.
\end{abstract}

\section{Introduction}

Medium Altitude Long Endurance aircrafts (MALEs) are designed to perform missions in a wide range of environments for a long period of time exposing the wing fuel to extreme ambient temperatures. The wings of such type of aircrafts 
are designed to obtain a high aerodynamic efficiency resulting in high aspect ratio wings (i.e. high area-to-volume ratio). Moreover there is no insulation between the external air and the wing fuel except the wing skin, which is reduced to the minimum for weight reason. All this factors cause a high heat loss/absorption of the wing fuel to/from the external ambient and could result in fuel temperatures out of operational limits.

The present paper describes a heat transfer dynamic model for an aircraft wing. The model is configured for a high aspect ratio wing made of carbon fibre but can be easily configured for others geometries and material properties. The model has been used to estimate the minimum, maximum and rate of change of the wing fuel temperature in the preliminary phase of an UAV design. The output of the dynamic simulation provides reliable data to validate the design against the requirements or to introduce modifications/limitation to fulfil them.

\section{Background}

In aviation, aircraft wings can be exposed to extreme cold ambient temperatures $\left(-70^{\circ} \mathrm{C}\right)$ during flight. Anti-ice additives are commonly used in aviation to permit flight in extreme cold environments. The table below shows the freezing point of some aviation fuels.

Table 1: Aviation fuel freezing points.

\begin{tabular}{|c|c|}
\hline Fuel & Freezing point (deg C) \\
\hline Jet A & -40 \\
\hline Jet A-1 & -47 \\
\hline Jet B & -50 \\
\hline
\end{tabular}

In civil aviation, normally flying at Mach $0.8-0.88$ with a high content of fuel, the problem of fuel freezing is not very common. However a fuel cold management is done, and consists of detecting a low limit temperature in the fuel and follows a warning procedure to avoid the fuel freezing (i.e. to descend to lower flight levels with hotter ambient temperature or increase the aircraft speed).

The problem is greater in UAV, for example, HALEs must maintain the high flight level during hours with a very low speed (i.e. $40 \mathrm{kft}$ and Mach 0.3 ), and therefore an alternative means of fuel cold management should be carried out for this type of aircraft.

On the other hand, for a hot environment, the temperature limit of the fuel is one that allows the correct fuel engine injection, and this could be up to $95^{\circ} \mathrm{C}$. The main causes that lead to a fuel heating are the use of it for engine oil cooling and the exposure to solar radiation in a warm environment. Typically, aircraft fuel systems have a fuel cooling subsystem to maintain the fuel within limits. The exposure of the wing to solar radiation in hot environments and aircraft on ground can lead to fuel temperatures up to $70^{\circ} \mathrm{C}$. This is not a critical problem if the fuel is not used for other purposes than as a power source. 
However, more and more, there is a high optimisation of the energy in aircrafts and the fuel is used as heat sink for the avionics cooling. In this case, the fuel should be maintained below a temperature to be effective as coolant. In flight this is achieved flying above a certain altitude to but on ground the problem is higher and in this case a possible solution is to water the wing to keep the fuel below a temperature.

The wing tank model estimates and analyses the fuel thermal behaviour against the different ambient and operational conditions. This permits the designer to search the best design solution.

\section{Model description}

\subsection{General description and assumptions}

This model estimates the fuel temperature inside the tank of an airplane wing. Although it has been used for studies of fuel absorption and heat loss in an UAV, it can also be used for any other aircraft type with minor changes to the input data and boundary conditions. The model has been developed in a dynamic simulation software tool called EcosimPro.

The model calculates the energy balance along time in the components of the thermal problem (i.e. fuel, internal air, wing spars and wing skin). A finite elements method is applied. The volume of fuel is split in ' $n$ ' finite elements along the wingspan. Each finite element interchanges energy with the upper wing skin and lower wing skin (i.e. ceiling and floor of tank), rear and front spars, air inside the tank and the inlet and outlet fuel enthalpies.

The calculation of heat transfer from the outside to the internal tank (i.e. to the fuel and internal air) through the wing skin is done by a finite element method. In this case the skin has ' $\mathrm{n}$ ' elements along the wingspan and ' $\mathrm{m}$ ' elements along the thickness of the skin. In a similar way the heat transfer from the leading edge and trailing edge to the tank through the front and rear spars is calculated. Thus each tank wall is defined by two-dimensional finite element (i,j).

The model has a geometry module that calculates all needed geometric data for the heat transfer modules. It calculates firstly the fuel level along the time and afterwards the contact area of the remaining fuel with the tank walls. It also provides other geometric parameters for the heat transfer (i.e. characteristic length, perimeter, etc.).

The solar radiation on the wing for each instant of the operation of the aircraft is calculates by a 'radiation' module. This module calculates the sun position and the actual radiation that reaches the wing (i.e. by subtracting the absorptivity of the atmosphere). The same module calculates the radiation from the surface of the wing to space.

The thermal and fluid properties (i.e. density, conductivity, Prandtl, viscosity, etc.) of the different fluids and solids involved in the problem (air, fuel and carbon fibre) are calculated in a specific module. 
Finally the model has a module for user inputs. These are of two types, constants such as fuel type, time, date and location of operation of the airplane, tank geometry, etc., and variables throughout the simulation such as altitude, speed and fuel consumption.

The figure below shows a block diagram with the relationship between the different modules.

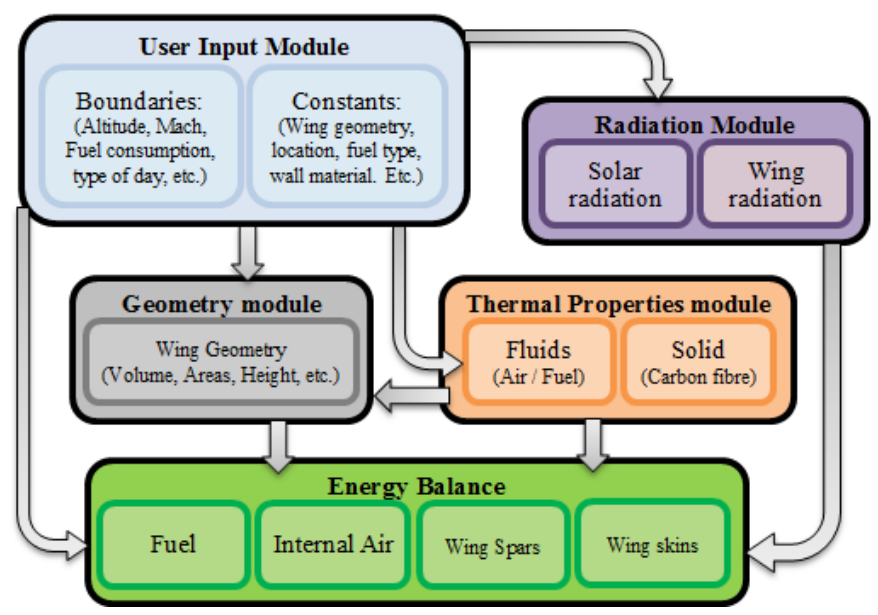

Figure 1: Wing fuel tank thermal model.

\subsection{Assumptions}

The following assumptions are listed:

- Standard atmosphere is considered for all ambient calculations.

- Cloudiness is not considered to have the maximum effect of solar radiation.

- Wing fuel tank has been considered as a trapezoidal volume. No internal structures (i.e. ribs) have been modelled.

- The attitude of the wing (i.e. dihedral, attack and balance angles) is constant and remains unchanged along the aircraft operation.

- The thickness of the wing is reduced linearly along the wingspan.

- No humidity in the air has been considered.

- JP8 fuel has been used.

- Carbon fibre is used for all the tank structures (skins and spars).

- Gray paint with an absorptivity of 0.9 has been used for the wing.

\subsection{Geometry}

This module finds the fuel level inside the tank along time. It also discretizes the fuel volume in finite element and calculates the corresponding fuel level of each one and the contact areas for heat transfer. The tank is defined by the upper and lower skins and the front and rear spars. The following figure shows the wing fuel tank geometry and the finite element discretization. 

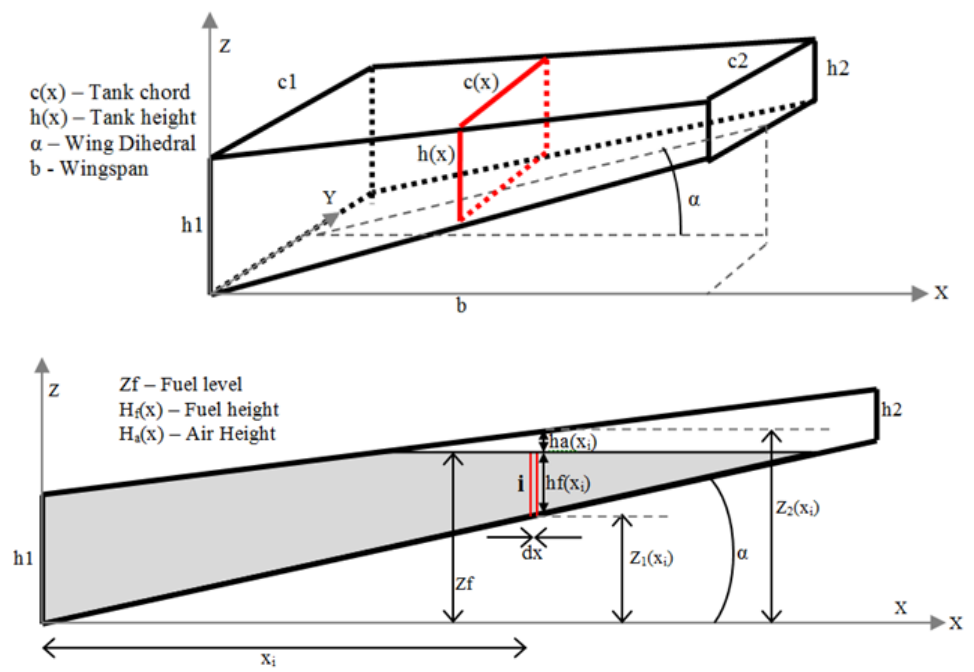

Figure 2: Wing fuel tank geometry.

The fuel height ' $h_{\mathrm{f}}$ ' for each finite element is:

$$
h_{f}\left(x_{i}\right)=\left\{\begin{array}{c}
Z_{f}-Z_{1}\left(x_{i}\right), Z_{1}\left(x_{i}\right)<Z_{f}<Z_{2}\left(x_{i}\right) \\
h\left(x_{i}\right), Z_{f}>Z_{2}\left(x_{i}\right) \\
0, Z_{f}<Z_{1}\left(x_{i}\right)
\end{array} .\right.
$$

The known variable is the total fuel volume in each time, in order to find $\mathrm{Zf}$, the model matches the total volume to the integration all the differential volumes.

$$
V_{f}=\int_{0}^{b} d V\left(Z_{f}, x\right) d x \Rightarrow Z_{f}
$$

The rest of geometry (i.e. contact areas for heat transfer) is derived from $\mathrm{h}_{\mathrm{f}}\left(\mathrm{x}_{\mathrm{i}}\right)$.

Finally this module also calculates the inlet and outlet mass flow in each control volume with $\frac{\partial z_{f}}{\partial t}$ value.

\subsection{Radiation}

This module calculates the radiation from the sun to the upper wing surface and radiation emitted (radiative cooling) from the surface to the clear sky. 


\section{Solar radiation}

The module firstly calculates the zenith, elevation and azimuth angles of the sun along the time in a specific location (i.e. longitude and latitude) based on equations from 'Astronomical Algorithms', by Meeus [6]. The sun position is corrected with the earth eccentricity along the years and atmospheric refraction effects. This permits to estimate an accuracy solar radiation during the flight of the aircraft.

With the zenith the model calculates the solar radiation with the equations proposed by Bird [1]. In order to find the actual radiation in the earth surface it calculates the transmittance of the atmosphere (gases, water vapour and ozone) with some specific user inputs (i.e. thickness of different atmosphere layers, ambient pressure, etc.) and applies it to the extra-terrestrial sun radiation. The extra-terrestrial sun radiation is also corrected with the variation of distance from sun to earth along the solar year. Ground and atmospheric albedo is not considered to experience the maximum radiation.

$$
I_{T}=I_{o} \cos (z) K_{d}+I_{o} \cos (z) K_{a s}
$$

$\mathrm{I}_{\mathrm{o}}$ : Extra-terrestrial solar irradiance corrected with the distance earth-sun factor

$\mathrm{I}_{\mathrm{T}}$ : Total solar irradiance on a horizontal surface

$\mathrm{K}_{\mathrm{d}}$ : Correction factor of atmosphere layers absorptivity for direct irradiance.

$\mathrm{K}_{\mathrm{as}}$ : Correction factor of atmosphere layers absorptivity for scattering irradiance.

\section{Radiative cooling:}

The upper surface of the wing is radiating heat to the sky in the form of longwaves. This radiation is calculated with the parameterisation of clear sky emissivity proposed by Berdahl and Martin [3].

$$
\begin{gathered}
Q_{e m m}=\varepsilon_{\text {surface }} \sigma\left[T_{\text {surface }}^{4}-T_{\text {sky }}^{4}\right] . \\
T_{\text {sky }}^{4}=\varepsilon_{\text {sky }} T_{a m b}^{4} . \\
\varepsilon_{\text {sky }}=0.711+\frac{56}{10^{4}} T_{d}+\frac{73}{10^{6}} T_{d}^{2}+\frac{13}{10^{3}} \cos (2 \pi H / 24)+\frac{12}{10^{5}}(p-1000) .
\end{gathered}
$$

$\mathrm{T}_{\mathrm{d}}$ : $\quad$ Dew point temperature in Celsius

$\mathrm{T}_{\mathrm{amb}}$ : Ambient temperature

$\mathrm{H}: \quad$ Solar time in hour

p: $\quad$ Ambient Pressure in mbar

The lower surface of the wing also exchanges heat by radiation with the land surface. This effect has not been considered in the current model due to the complexity of estimating the land temperature for different locations of the earth (i.e. from -25 to $40^{\circ} \mathrm{C}$ ). This is an improvement for the next model version. 


\subsection{Energy balance}

\subsubsection{Fuel energy balance}

This module calculates the fuel temperature over time using the energy balance equation in each finite fuel element. Heat is interchanged with the upper and lower wing tank surfaces, the rear and front spars, and the air inside the tank. Additionally an inlet and outlet of energy in form of fuel enthalpy from/to the adjacent fuel finite elements is considered.

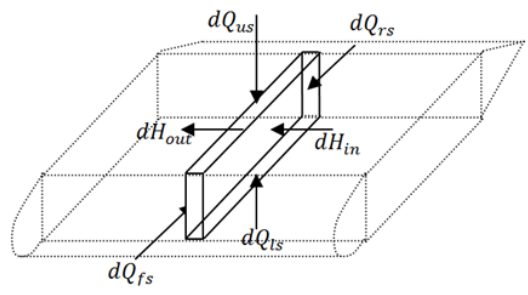

Figure 3: Fuel Energy balance.

$$
\begin{gathered}
\frac{\partial\left[T_{f}\left(x_{i}\right) m_{f}\left(x_{i}\right) c_{p}\left(x_{i}\right)\right]}{\partial t}=d W_{\text {out }}\left(x_{i}\right)-d Q_{l s}\left(x_{i}\right)-d Q_{u s}\left(x_{i}\right), \\
-d Q_{f s}\left(x_{i}\right)-d Q_{r s}\left(x_{i}\right)-d Q_{a}\left(x_{i}\right)+d H_{\text {in }}\left(x_{i}\right)-d H_{\text {out }}\left(x_{i}\right) .
\end{gathered}
$$

The heat exchanged with the lower skin of the wing 'ls' is calculated with the natural convection equations for a horizontal plate. The Nussel number in this case depends on whether the fuel is hotter or colder that the lower surface. When the lower skin is hotter than fuel (unstable situation) the equations used are those proposed by Raithby and Hollands [7]. On the other hand when the lower skin is colder than fuel (stable situation) the equations used are the proposed by Fujii and Imura [8].

$$
\begin{gathered}
d Q_{l s}\left(x_{i}\right)=H_{l s}\left(x_{i}\right) C\left(x_{i}\right) d x\left[T_{f}\left(x_{i}\right)-T_{l s}\left(x_{i}\right)\right] \text { if } h_{f}\left(x_{i}\right)>0 . \\
H_{l s}\left(x_{i}\right)=\frac{N u\left(x_{i}\right) K_{f}\left(x_{i}\right)}{C\left(x_{i}\right)} . \\
N u=\left\{\begin{array}{c}
\frac{0.560 R a^{1 / 4}}{\left(1+(0.492 / P r)^{9 / 16}\right)^{4 / 9}}, R a<10^{7} \\
0.14 R a^{1 / 3}\left(\frac{1+0.0107 P r}{1+0.01 P r}\right),
\end{array}\right. \text { for 'ls' hotter than Fuel. } \\
N u=0.58 R a^{1 / 5} \text { for 'ls'colder than Fuel. }
\end{gathered}
$$


The heat exchanged with the upper skin of the wing 'us' is also calculated with the natural convection equations for a horizontal plate. This heat is only calculated when there exists a contact between the tank surface and the fuel. The same equations as per lower skin are applicable, but exchanged.

$$
d Q_{u s}\left(x_{i}\right)=H_{u s}\left(x_{i}\right) C\left(x_{i}\right) d x\left[T_{f}\left(x_{i}\right)-T_{u s}\left(x_{i}\right)\right] \text { if } h_{f}\left(x_{i}\right)=h\left(x_{i}\right) .
$$

The heat exchanged with the front and rear spars, 'fs' and 'rs' respectively, is calculated with the natural convection equations for a vertical plate. The nussel numbers is calculated with equation proposed by Churchill and Chu.

$$
\begin{aligned}
& d Q_{f s}=H_{f s}\left(x_{i}\right) h_{f}\left(x_{i}\right) d x\left[T_{f}\left(x_{i}\right)-T_{f s}\left(x_{i}\right)\right] . \\
& d Q_{r s}=H_{r s}\left(x_{i}\right) h_{f}\left(x_{i}\right) d x\left[T_{f}\left(x_{i}\right)-T_{r s}\left(x_{i}\right)\right] . \\
& N u=0.68+0.67 R^{1 / 4}\left[1+\left(\frac{0.492}{P r}\right)^{9 / 16}\right]^{-4 / 9} .
\end{aligned}
$$

The heat exchanged with the internal air ' $a$ ' of the tank is calculated with the natural convection equations for a horizontal plate. In this case for the Nussel number calculation, the same equation as per heat transfer between fuel and lower skin has been used, considering now the fuel as the lower skin. This heat is only calculated when exists air above the fuel (i.e. the fuel level is below the upper skin).

$$
\mathrm{dQ}_{\mathrm{a}}\left(\mathrm{x}_{\mathrm{i}}\right)=\mathrm{H}_{\mathrm{a}}\left(\mathrm{x}_{\mathrm{i}}\right) \mathrm{C}\left(\mathrm{x}_{\mathrm{i}}\right) \mathrm{dx}\left[\mathrm{T}_{\mathrm{f}}\left(\mathrm{x}_{\mathrm{i}}\right)-\mathrm{T}_{\mathrm{a}}\right] \text { if } 0<h_{\mathrm{f}}\left(\mathrm{x}_{\mathrm{i}}\right)<h\left(\mathrm{x}_{\mathrm{i}}\right) .
$$

Finally, the inlet and outlet enthalpies in the control volume are the following:

$$
\begin{array}{r}
\mathrm{dH}_{\mathrm{in}}\left(\mathrm{x}_{\mathrm{i}}\right)=\mathrm{c}_{\mathrm{p}}\left(\mathrm{x}_{\mathrm{i}+1}\right) \dot{\mathrm{m}}_{\mathrm{f}_{-} \text {in }}\left(\mathrm{x}_{\mathrm{i}}\right)\left[\mathrm{T}_{\mathrm{f}}\left(\mathrm{x}_{\mathrm{i}+1}\right)\right] . \\
\mathrm{dH}_{\text {out }}\left(\mathrm{x}_{\mathrm{i}}\right)=\mathrm{c}_{\mathrm{p}}\left(\mathrm{x}_{\mathrm{i}}\right) \dot{\mathrm{m}}_{\mathrm{f}_{-} \text {out }}\left(\mathrm{x}_{\mathrm{i}}\right)\left[\mathrm{T}_{\mathrm{f}}\left(\mathrm{x}_{\mathrm{i}}\right)\right] .
\end{array}
$$

\subsubsection{Internal air}

The energy balance of the internal air considers it as an ideal gas and applies the energy equation to a unique volume of air. It considers all the heat from/to walls and fuel, and the enthalpy of the flow from/to external to maintain the tank at ambient pressure.

\subsubsection{Wing skins and spars energy balance}

To calculate the energy balance in the tank walls, these are divided into twodimensional finite elements. The dimension ' $i$ ' corresponds to ' $n$ ' elements in the direction of the wingspan, while the dimension ' $j$ ' corresponds to ' $m$ ' elements 
in the direction of the wall thickness. The division of the wall finite elements (i) in the direction of the wingspan matches with the fuel finite elements (i). The following picture shows a finite element of the upper skin. The rest of walls are split in a similar way.

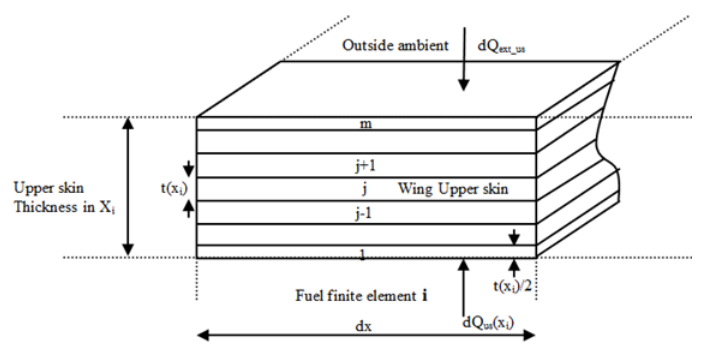

Figure 4: Finite element of the wall.

Applying the energy balance to the ' $\mathrm{i}$ ' finite element of the upper skin we have:

- Node 1 (inner element of the wall)

$$
\begin{gathered}
c_{f} \rho_{f} d x C\left(x_{i}\right) \frac{t\left(x_{i}\right)}{2} \frac{\partial T_{u s}(i, 1)}{\partial t} \\
=d Q_{u s}\left(x_{i}\right)+\frac{k_{f}}{t\left(x_{i}\right)} d x C\left(x_{i}\right)\left[T_{u s}(i, 2)-T_{u s}(i, 1)\right], \\
+\frac{k_{f}}{d x} \frac{t\left(x_{i}\right)}{2} C\left(x_{i}\right)\left[T_{u s}(i+1,1)-2 T_{u s}(i, 1)+T_{u s}(i-1,1)\right] .
\end{gathered}
$$

- Node $\mathrm{j}$

$$
\begin{gathered}
c_{f} \rho_{f} d x C\left(x_{i}\right) t\left(x_{i}\right) \frac{\partial T_{u s}(i, j)}{\partial t} \\
=\frac{k_{f}}{t\left(x_{i}\right)} d x C\left(x_{i}\right)\left[T_{u s}(i, j+1)-2 T_{u s}(i, j)+T_{u s}(i, j-1)\right] \\
+\frac{k_{f}}{d x} \frac{t\left(x_{i}\right)}{2} C\left(x_{i}\right)\left[T_{u s}(i+1, j)-2 T_{u s}(i, j)+T_{u s}(i-1, j)\right] .
\end{gathered}
$$

- Node m (outer element of the wall)

$$
\begin{gathered}
c_{f} \rho_{f} d x C\left(x_{i}\right) \frac{t\left(x_{i}\right)}{2} \frac{\partial T_{u s}(i, m)}{\partial t}=d Q_{\text {ext }}\left(x_{i}\right) \\
+\frac{k_{f}}{t\left(x_{i}\right)} d x C\left(x_{i}\right)\left[T_{u s}(i, m-1)-T_{u s}(i, m)\right] \\
+\frac{k_{f}}{d x} \frac{t\left(x_{i}\right)}{2} C\left(x_{i}\right)\left[T_{u s}(i+1, m)-2 T_{u s}(i, 1)+T_{u s}(i-1, m)\right] .
\end{gathered}
$$


Similar equations are used for the energy balance of the lower skin (ls), front spar (fs) and rear spar (rs). Finally, the external heat in the outer elements of the walls is calculated as follows.

For the upper skin, the sum of the solar radiation, the emitted radiation from the surface to the clear sky and the convection with the external air (forced or natural depending on the airspeed).

$$
d Q_{\text {ext_us }}\left(x_{i}\right)=d Q_{\text {radiation }}\left(x_{i}\right)-d Q_{\text {emmited }}\left(x_{i}\right)+d Q_{\text {Convection }}\left(x_{i}\right) \text {. }
$$

The solar radiation and heat emitted to the sky were calculated in the 'radiation' module. The convection term is calculated as follows.

$$
d Q_{\text {Convection_us }}\left(x_{i}\right)=\mathrm{H}_{\text {ext_us }}\left(\mathrm{x}_{\mathrm{i}}\right) \mathrm{C}\left(\mathrm{x}_{\mathrm{i}}\right) \mathrm{dx}\left[\mathrm{T}_{\text {air_recovery }}\left(\mathrm{x}_{\mathrm{i}}\right)-\mathrm{T}_{\mathrm{us}}\left(\mathrm{x}_{\mathrm{i}}\right)\right] .
$$

For forced convection (i.e. airspeed above $0.5 \mathrm{~m} / \mathrm{s}$ ) the nussel number is calculated based on the equation proposed by Churchill:

$$
\begin{gathered}
N u=0.45+\left(0.6774 \phi^{1 / 2}\right)\left(1+\frac{(\phi / 12500)^{3 / 5}}{\left(1+\left(\phi_{u m} / \phi\right)^{7 / 2}\right)^{2 / 5}}\right)^{1 / 2} . \\
\phi=\operatorname{RePr}^{2 / 3}\left(1+(0.0468 / P r)^{2 / 3}\right)^{1 / 2}, \phi_{u m}=1.875 \phi\left(\operatorname{Re}=\operatorname{Re}_{u}\right) . \\
R e_{u} \cong \text { Reynolds of transition from laminar flow to turbulent }
\end{gathered}
$$

For natural convection (i.e. airspeed below $0.5 \mathrm{~m} / \mathrm{s}$ ) the equations used for nussel calculation are the same as the heat transfer between the fuel and the lower skin (horizontal plate), but now the fluid is the ambient air and the plate is the upper skin.

The external heat transfer for the lower skin is the convection (forced or natural) between the horizontal lower skin and the external air (i.e. horizontal plate natural convection equations are used). The external heat transfer for the front and rear spar is the natural convection between the vertical spar and the air inside the leading and trailing edge of the wing. (i.e. vertical plate natural convection equations are used)

\subsection{Thermal properties}

This module calculates all the thermal properties of the solids and fluids involved in the problem. The following table shows the input and outputs of this module. Data are extracted from Fuel handbooks and different materials literature. 
Table 2: Thermal properties data.

\begin{tabular}{|c|c|c|}
\hline Air & Inputs & Outputs \\
\hline Fuel & Temp. \& Pressure & $\begin{array}{c}\text { Density, thermal Conductivity, Specific Heat, } \\
\text { thermal diffusivity, thermal expansion, cinematic } \\
\text { viscosity and prandtl number. }\end{array}$ \\
\hline Carbon Fibre & None & $\begin{array}{c}\text { Density, thermal Conductivity, Specific Heat, } \\
\text { Density, thermal diffusivity, thermal expansion, } \\
\text { cinematic viscosity and prandtl number. }\end{array}$ \\
Conductivity, Specific Heat, Density and \\
Absorptivity.
\end{tabular}

\subsection{User input module}

This module gathers and manages all the inputs from the user. Some of them are used by the same module to calculate other needed variables, for example the ambient temperature and pressure in terms of the altitude. The user has to introduce the required constants and the problem boundaries along the time.

Table 3: User inputs for the model.

\begin{tabular}{|c|c|c|}
\hline & Inputs & Outputs \\
\hline 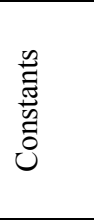 & $\begin{array}{l}\text { Number of finite elements ' } \mathrm{i} \text { ' and ' } \mathrm{j} \text { ' } \\
\text { Tank geometry } \\
\text { Initial Fuel Mass } \\
\text { Wing Dihedral angle and Wingspan } \\
\text { Location and date data } \\
\text { Radiation data }\end{array}$ & \\
\hline$\stackrel{\Xi}{\stackrel{0}{\ominus}}$ & $\begin{array}{l}\text { Altitude }(\mathrm{t}) \text {, Mach }(\mathrm{t}) \text {, Wing Fuel } \\
\text { consumption }(\mathrm{t}) \\
\text { Relative humidity }(\mathrm{t})\end{array}$ & $\begin{array}{l}\text { Ambient Pressure, and } \\
\text { temperature, Recovery } \\
\text { temperature, Airspeed }\end{array}$ \\
\hline
\end{tabular}

\section{Simulation results}

To illustrate this some simulations have been ran to check the thermal behaviour of the fuel, walls and internal air of a wing fuel tank when performing some typical UAV missions in hot, cold and warm ambient. The following plots show the flight profile, fuel contents and main component temperature for each mission. The location, initial temperature of components and the starting time of the mission have been selected to simulate the worst condition from a thermal point of view. The defueling sequence of the wing tank starts around seven hours after the beginning of the mission. Two hours of soak previous to operation is preformed and an additional one hour of on ground operation with engine running. 


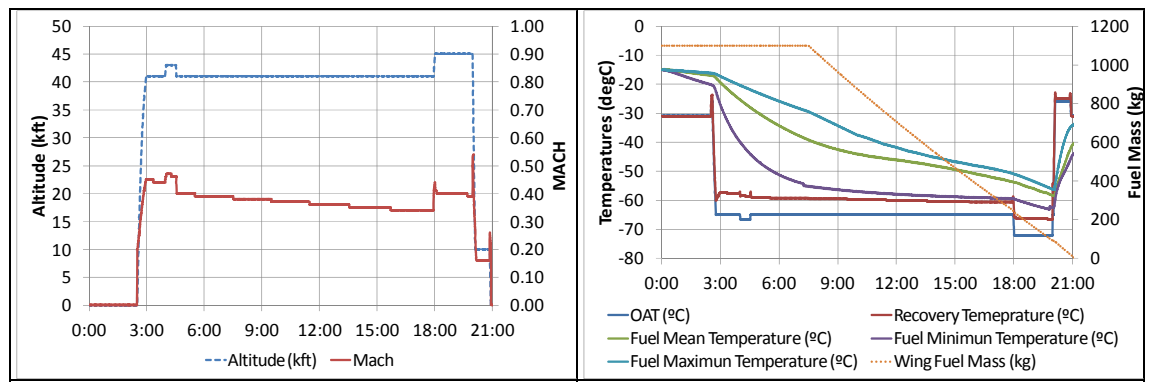

Figure 5: High altitude mission and cold environment (Moscow 25/12/2014).

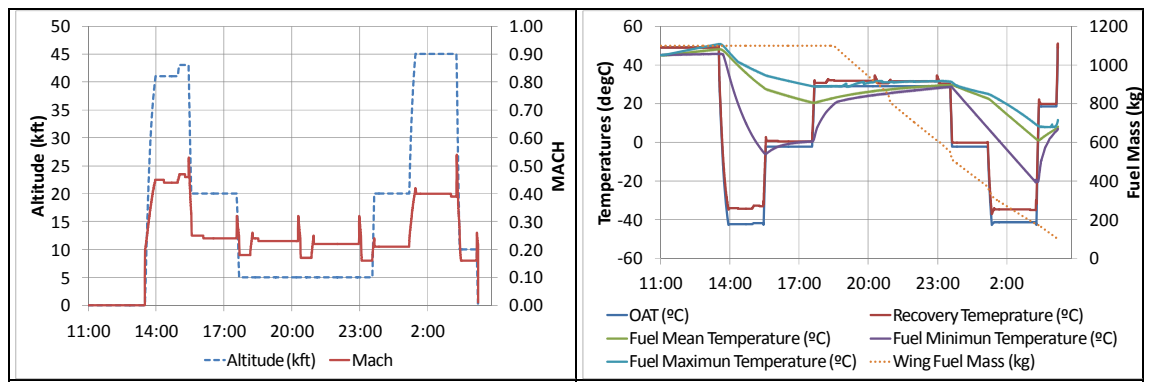

Figure 6: Low level mission and hot environment (Dubai 21/07/2014).

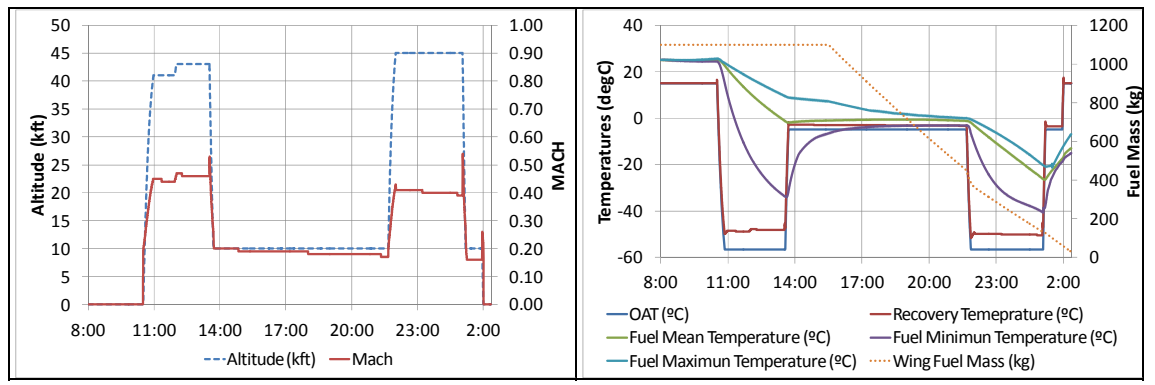

Figure 7: Medium level mission and warm environment (Madrid 13/03/2014).

For the cold environments, UAVs with a requirement of flying at a high altitude and low speeds which have high aspect ratio wings will experience temperatures below the freezing points. The time to reach the low limit depends on the fuel content (i.e. defueling sequence). It is essential to find solutions to perform this type of mission avoiding any fuel freezing. Possible solutions include consuming the wing fuel firstly, the use of heaters and the improving of insulation. 
With respect to the opposite environment (i.e. hot climates), the soak of the aircraft in the beginning of the mission increases considerably the wing skin temperature and therefore the fuel temperature. With regards to the temperatures during flight, they are always maintained below $50^{\circ} \mathrm{C}$.

\section{Conclusions}

It can be conclude that this simulation tool provides useful information to a fuel system designer. Using the tool it is possible to analyse the thermal behaviour of a wing tank's fuel, not only to detect problems of fuel freezing but also to analyse the fuel capability as coolant source.

\section{Acknowledgement}

The author of this paper would like to acknowledge and thank to all the Environmental and Fluids system department people of Airbus Defense and Space (Spain) for its knowledge and help to develop the model.

\section{References}

[1] Richard E. Bird \& Roland L. Hulstrom, A simplified clear sky model for direct and diffuse insolation on horizontal surfaces, Colorado, February 1981.

[2] John H. Lienhard IV/John H. Lienhard V, A heat Transfer Book text book Third Edition, Cambridge Massachusetts, 2003.

[3] Berdahl, P., Martin, M., 1984. Emissivity of clear skies. Solar Energy 32, 663-664.

[4] NOAA. Earth System Research Laboratory. http://www.esrl.noaa.gov/

[5] Frank M. White, Fluid Mechanics. McGraw-Hill, Inc., USA.

[6] Meeus, J., 1998. Astronomical Algorithms, second ed. Willmann-Bell, Inc., Richmond, Virginia, USA.

[7] G. D. Raithby \& K. G. T. Hollands. Natural convection. In W. M. Rohsenow, J. P. Hartnett \& Y. I. Cho, editors, Handbook of Heat Transfer, chapter 4. McGraw-Hill, New York, 3rd edition, 1998.

[8] T. Fujii and H. Imura. Natural convection heat transfer from a plate with arbitrary inclination. Int. J. Heat Mass Transfer, 15(4): 755-767, 1972. 